\title{
The Zwicky Transient Facility Observing System
}

\author{
Roger M. Smith ${ }^{1} *$, Richard G. Dekany ${ }^{1}$, Christopher Bebek ${ }^{2}$, Eric Bellm ${ }^{1}$, Khanh Bui ${ }^{1}$, John \\ Cromer ${ }^{1}$, Paul Gardner ${ }^{1}$, Matthew Hoff ${ }^{2}$, Stephen Kaye ${ }^{1}$, Shrinivas Kulkarni ${ }^{1}$, Andrew Lambert ${ }^{2}$, \\ Michael Levi ${ }^{2}$ and Dan Reiley ${ }^{1}$
}

\author{
${ }^{1}$ Caltech Optical Observatories, 1200 E. California Blvd, CA 91125, USA \\ ${ }^{2}$ Lawrence Berkeley National Labs, 1 Cyclotron Road, Berkeley, CA 94720, USA
}

\begin{abstract}
The Zwicky Transient Facility (ZTF) is a synoptic optical survey for high-cadence time-domain astronomy. Building upon the experience and infrastructure of the highly successful Palomar Transient Factory (PTF) team, ZTF will survey more than an order of magnitude faster than PTF in sky area and volume in order to identify rare, rapidly varying optical sources. These sources will include a trove of supernovae, exotic explosive transients, unusual stellar variables, compact binaries, active galactic nuclei, and asteroids. The single-visit depth of $20.4 \mathrm{mag}$ is well matched to spectroscopic follow-up observations, while the co-added images will provide wide sky coverage $1.5-2$ mag deeper than SDSS. The ZTF survey will cover the entire Northern Sky and revisit fields on timescales of a few hours, providing hundreds of visits per field each year, an unprecedented cadence, as required to detect fast transients and variability. This high-cadence survey is enabled by an observing system based on a new camera having $47 \mathrm{deg}^{2}$ field of view - a factor of 6.5 greater than the existing PTF camera - equipped with fast readout electronics, a large, fast exposure shutter, faster telescope and dome drives, and various measures to optimize delivered image quality. Our project has already received an initial procurement of e $2 \mathrm{v}$ wafer-scale CCDs and we are currently fabricating the camera cryostat. International partners and the NSF committed funds in June 2014 so construction can proceed as planned to commence engineering commissioning in 2016 and begin operations in 2017. Public release will allow broad utilization of these data by the US astronomical community. ZTF will also promote the development of transient and variable science methods in preparation for the seminal first light of LSST.
\end{abstract}

Keywords: $\mathrm{CCD}$, mosaic, wide-field, digital correlated double sampling, concurrent clocking, differential signal path, Schmidt telescope, CCD231-C6

\section{INTRODUCTION}

The Zwicky Transient Facility (ZTF) will be a significant new capability for exploring the transient universe at high cadence (frequent revisits). Building on the existing Palomar Transient Factory (PTF) and intermediate PTF (iPTF) infrastructure at Palomar Mountain, ZTF consists of both an Observing System (OS) and a significant new Data System (DS). In this paper, we shall describe the ZTF OS. ZTF DS will be described in future publications.

The ZTF OS consists of a new ZTF Camera plus significant modifications to the $1.2 \mathrm{~m}$ aperture Samuel Oschin Telescope to enable the vastly increased survey speed demanded for ZTF. The main mission for the ZTF OS will be to scan large areas of the available sky several times per night at 1 arcsec pixel sampling ( $2 \operatorname{arcsec}$ FWHM) to search for transient events on much shorter time scales than can be efficiently detected with the present iPTF[1]. In addition, the multiple visits, averaging nearly 300 epochs at each location in the Northern Hemisphere per year, will be co-added to create a deep two-color survey of a large area of the sky with particular emphasis on the Northern declination cap to allow source selection for DESI[2].

*rsmith@astro.calteche.edu, (626) 395-8780. SPIE 9154, Montreal 2014. 
ZTF prioritizes field of view over depth to bias transient event detection towards targets that are bright enough for follow-up spectroscopy, a fundamental difference and complimentary function to LSST. The $1.2 \mathrm{~m}$ Oschin Schmidt Telescope will be upgraded with a new camera that provides 6.5 times the instantaneous field of view of the present PTF (Figure 1).

For $30 \mathrm{~s}$ exposures during dark time, the limiting magnitude is expected to be 21.5 in Sloan g' and 21 in Mould R. Reducing overheads from $46 \mathrm{~s}$ (in PTF) to less than $15 \mathrm{~s} \mathrm{(10} \mathrm{s} \mathrm{goal),} \mathrm{through} \mathrm{faster} \mathrm{CCD} \mathrm{readout} \mathrm{and} \mathrm{telescope} \mathrm{and} \mathrm{dome}$ drive upgrades, allows optimal exposure time to be reduced from $60 \mathrm{~s}$ to $30 \mathrm{~s}$ to improve frame rate by 2.7 ( $106 \mathrm{~s}$ frame time $\rightarrow 40 \mathrm{~s})$ and duty cycle by $18 \%(57 \%$ duty cycle $\rightarrow 75 \%)$, while remaining sky noise limited (darkest sky $>25$ e/s/pix).

At $386 \mathrm{~mm} \times 395 \mathrm{~mm}$ corner to corner and $89 \%$ fill factor, the CCD mosaic has $8 \%$ greater field of view than the 14 inch photographic plates used on the same telescope during the two color Palomar Optical all Sky Survey from 1950 to 1957, POSS [3]. The fully automated ZTF can observe the same number of square degrees as POSS $(33,660)$ to similar depth, in just 8 hours per color. Images will be relayed in near real time to the Caltech's IPAC, where they will be processed and compared automatically to detect new transients within minutes of the latest observation. At a slower rate, IPAC will process fully calibrated data and house a legacy archive of all ZTF data.

Many improvements, such as autoguiding, autofocusing, active tilt and collimation, and removal of heat from the dome, are aimed at delivering 2 arcsecond image quality (with 1 arcsec/pixel sampling) more consistently than for the present PTF survey. Higher CCD QE in the bandpasses of interest will also enhance throughput. Dewar, shutter, filter and spiders are being redesigned to limit beam obstruction to only $22.5 \%$ (compared to $19 \%$ for PTF), in spite of the large increase in field of view.

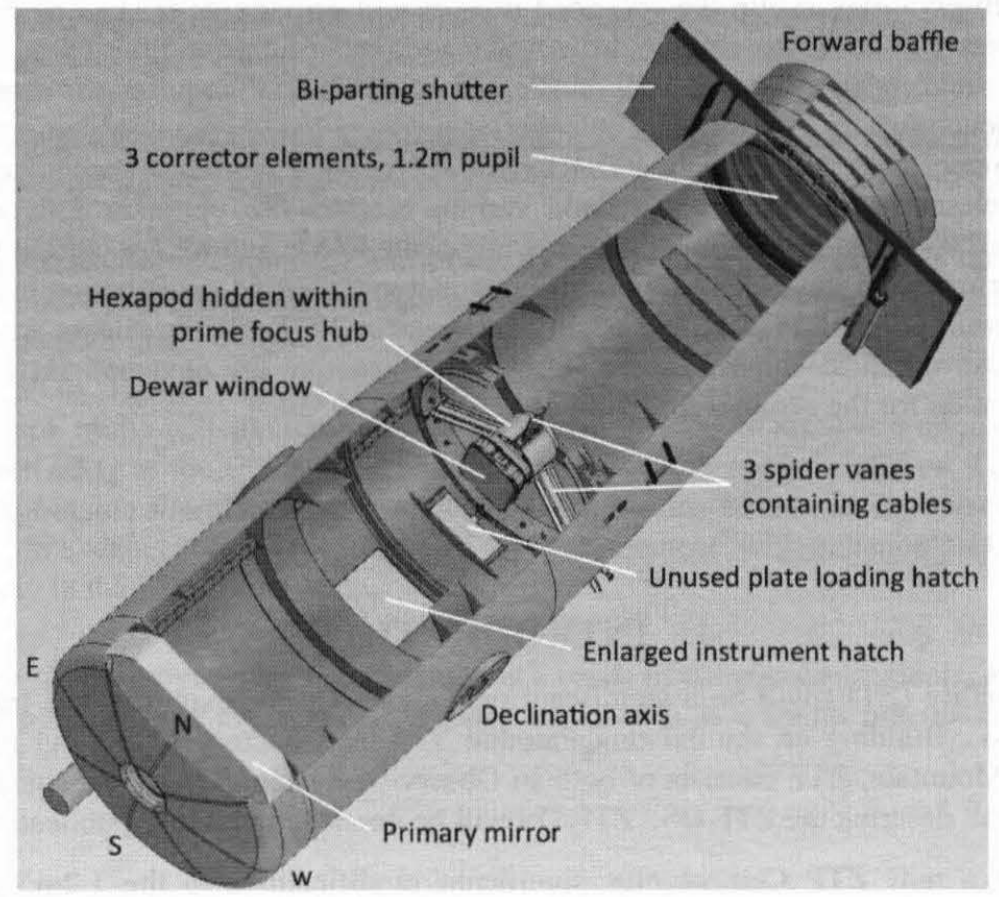

Figure 1: Oschin 48" Schmidt Telescope showing the ZTF camera installed on new 3 vane spiders and hexapod.

\section{SCIENCE CCDS}

CCD manufacturers have reduced defect density to the point where lowest cost per unit area is achieved with the largest CCD that fits on a $150 \mathrm{~mm}$ silicon wafer. In 2012 ZTF executed a competitive bid process for the sixteen $6144 \times 6160$ pixel CCDs required to completely tile a $47 \mathrm{deg}^{2}$ instantaneous field of view, selecting e2v, Inc. in February 2013. The anticipated high yield has been confirmed by the delivery of the first batch of six CCD231-C6s within 9 months after placement of the order. These met all specifications, and exceeded them in key areas such as height, flatness, QE, and cosmetic defects. 
Fortuitously, the standard $15 \mu \mathrm{m}$ pixel size maps to 1 arcsec providing Nyquist sampling of the anticipated 2 arcsec delivered image quality. This image sampling matches that of PTF, providing adequate spatial sampling while minimizing the number of pixels to be digitized, stored and processed. By sampling the PSF no more finely than Nyquist, not only is the number of pixels minimized but signal per pixel is maximized, relaxing read noise requirement so that pixel rate can be higher. Substantial cost savings are then realized by meeting the 10 s readout goal with only four outputs per CCD. The single-ended read noise measured by e $2 \mathrm{v}$ at $500 \mathrm{kHz}$ for the 24 output amplifiers delivered so far shows a tight distribution: allowing $\sqrt{2}$ noise increase for differential outputs and another $\sqrt{2}$ for $1 \mathrm{MHz}$ operation, the mean read noise is projected to be $11.1 \mathrm{e}$ - with $0.3 \mathrm{e}$ - standard deviation. This degrades total noise by $9 \%$ for $30 \mathrm{~s}$ exposures in darkest sky conditions.

The only customization of the CCD231-C6 is the single layer AR coating thickness. QE averaged over all pixels received to date is $82 \%$ at $400 \mathrm{~nm}$ rising to $97 \%$ at $500 \mathrm{~nm}$ and falling only to $80 \%$ at $700 \mathrm{~nm}$. Improvements at the band edges may be obtained on the remaining CCDs by employing some variant of the multilayer coating employed on identically sized CCD290-99s for JPAS[9].

The coarse image scale requires a CCD with the lowest possible lateral charge diffusion to minimize this contribution to PSF degradation. Since ZTF has no requirement for extended red response, a CCD with conventional thickness and resistivity could be selected. Not only does this improve yield and thus lower the cost, but also has the benefit that lateral charge diffusion can be reduced to 0.65 pixel FWHM in g' band and 0.45 pixel in R, merely by operating with positively biased image-area clocks to minimize undepleted thickness[4].

\section{CCD ELECTRONICS}

Commercial CCD controllers meeting both technical and schedule requirements for ZTF are available from both Astronomical Research Cameras (ARC) and Semiconductor Technology Associates (STA). The ARC "Gen-3" controller when equipped with double fiber transmitter, and the STA "Archon"[5] can both drive 4 CCDs from a single chassis. At the time of writing, preliminary selection of the "Archon" had been made with the full purchase being subject to the outcome of data transfer reliability and performance tests for the first unit. The Archon is preferred since the high speed digital correlated double sampling video chain has already been demonstrated and there are several packaging improvements. Board replacement is easier: cabling to the CCDs can be more easily and safely disengaged with minimal ESD risk to the CCDs, and boards are conductively cooled by direct mechanical coupling to an internal water-cooled heat sink.

To minimize the cost and complexity of the electronics a single controller operates each quadrant of the mosaic. A fifth controller runs the three extra focal imaging CCDs and one guider. Readout will be strictly pixel synchronous to avoid patterns in the images caused by clock-to-video crosstalk: all controllers share a common master clock, execute identical code and will be triggered by a common signal. This well-established modular approach is used in most large mosaics (eg. NOAO mosaics, PTF, PanSTARRS, DES, LSST.)

The clocks for four CCDs are ganged together so that each quadrant appears to be a single 16 channel CCD. The ganging of the same parallel clock across 4 CCDs leads to $\sim 275 \mu$ s minimum parallel shift time, given the $250 \mathrm{~mA}$ clock drive capability, and thus $0.85 \mathrm{~s}$ for the 3080 parallel shifts per frame.

Each serial clock driver is connected to the same pin on pairs of CCDs to provide 44ns worst-case transition time. Given the requirement for three coincident transitions, $1 \mathrm{MHz}$ pixel rate is easily supported.

The CCD electronics will be located outside telescope tube to avoid obstructing the beam. In the STA controller, the circuit boards are cooled by conduction to water cooled heat sinks within each chassis. Cooling water will be refrigerated to only slightly below ambient to avoid both condensation and the need for anti-freeze, which can be corrosive.

Total wiring length from CCD to electronics will be less than $1.5 \mathrm{~m}$, with cables hidden within the center of redesigned spider vanes. Propagation of CCD clocks along this length of cable is not expected to be a problem with the exception of the 100ns ResetGate pulse. This will be generated by a "pin driver" at the warm end of the CCD flex cable (within the dewar) so that only a low noise DC voltage needs to be supplied: the high frequency current will be supplied by bypass capacitors. The ResetGate control signal will be conveyed on twinax cable driven by a Low Voltage Differential Signal (LVDS) capable of $655 \mathrm{Mb} / \mathrm{s}$ up to $10 \mathrm{~m}$. 
For high channel count systems operating at high pixel rates, crosstalk and interference typically prove problematic. These effects will be suppressed by several orders of magnitude by operating the CCD231-C6 in true differential output mode. A dummy serial register and output amplifier also mimics the bias noise and clock feedthroughs. A differential preamplifier, located in vacuum at the warm end of the CCD's flex-cable, will drive a balanced twin-axial cable to a differential receiver and A/D converter in the electronics chassis. The differential receiver near the AD converter not only rejects common mode noise and crosstalk, but also suppresses the reset and clock feedthroughs that appear equally on signal and reference sides so that higher pixel rate can be supported.[10]

To support the high pixel rate, the typical analog dual slope integration is replaced by Correlated Double Sampling in the digital domain[6] as in PANSTARRS[7] and JPAS[8][9] mosaics. No analog processing is required beyond AC coupling and preamplification. A $100 \mathrm{MHz} 16$ bit differential ADC heavily oversamples the signal allowing for anti-aliasing at high enough frequency to provide negligible pixel-to-pixel memory. Averaging and subtraction of multiple samples is performed in Field Programmable Gate Arrays within the video cards to reduce noise. For a conventional analog CDS processor, gain would have to be set to $5 \mathrm{e}-/ \mathrm{ADU}$ so that 300,000 electron well capacity would use $90 \%$ of the 16 bit $\mathrm{ADC}$ range. The anticipated $23 \mathrm{e}-$ minimum shot noise from sky, then spans less than $5 \mathrm{ADU}$, and 10 e- read noise only spans 2 ADU. In the overscan, there is only read noise, which is not larger enough to adequately smooth ADC differential non-linearity. The Digital CDS solves this problem by operating at much higher bandwidth so that electronic noise dithers the samples over a larger number of ADC values. Averaging then interpolates to provide resolution in excess of 16 bits while smoothing the ADC Differential Non-Linearity and quantization errors[11].

\section{CAMERA SOFTWARE AND DATA PATH}

Each controller has a private GigaBit Ethernet link to a separate host computer. This link has ample bandwidth to carry signed 32 bit integers from all 16 channels at $1 \mathrm{MHz}$. While 32 bits is more than required to support oversampling of the read noise, using this format eliminates the need to unpack the data before lossless compression and writing to disk in FITS "Tile Compression" format. Each of the host computers is connected to a common network used to downlink the data to the processing facility at IPAC. About two thirds of the daily capacity of the current HPWREN (155 Mb/s) data link would be consumed to transmit 10 hours of observations. At $<1 \mathrm{~TB} /$ night for the whole mosaic, sufficient disk space can easily be distributed across the multiple host computers to hold several weeks of data in the event of downlink outages or traffic congestion.

One FITS file is created per CCD. Storage and downlink as separate files is most appropriate and simplest, since image processing typically occurs on a per CCD basis until multiple images are combined. Although the pixel data are compressed, headers are not, so images can be tracked without decompression.

\section{OPTICS}

Sensitivity is highly dependent on Delivered Image Quality (DIQ), since observations are sky noise dominated for both bandpasses, even in dark time. A detailed DIQ budget has been developed, which includes residual aberrations in the optical design, CCD charge diffusion, optical manufacturing and alignment tolerances, focal plane flatness/shape errors, focus and tilt sensing and control errors, seeing, atmospheric dispersion, telescope vibration induced by the wind or shutter, and tracking errors. (Table 1)

The Schmidt telescope was designed in the era of photography so the optical design did not account for a thick dewar window capable of resisting the 2.3 ton force exerted by the atmosphere. To achieve good image quality over all 16 CCDs ( $386 \mathrm{~mm} \times 395 \mathrm{~mm}$ corner to corner) in the presence of this dewar window, a $10 \%$ adjustment will be made to the aspheric coefficient of the Schmidt corrector assembly by refiguring an existing 50 -inch diameter blank and mounting it in front of the current cemented achromatic doublet to form an air-spaced triplet corrector. This strategy bypasses the need to refigure, or even reposition, the high-value 50-inch doublet. The optical design and fabrication tolerances on this zero-power optic have-been confirmed with potential vendors to be quite manageable, with lateral and axial positioning tolerances of the new plate of order $\pm 10 \mathrm{~mm}$.

Field curvature will be corrected by the combination of a slightly meniscus window (typ. $2739 \mathrm{~mm}$ radius concave on CCD side, typ. $5556 \mathrm{~mm}$ radius convex on exterior) with $28 \mathrm{~mm}$ center thickness, and by mounting CCDs on the chords of a $3062 \mathrm{~mm}$ radius sphere. An earlier optical design concept utilizing a plano-convex window was set aside in mid2014 in favor of the optical performance of the meniscus design. Individual field-flattening lenses mounted to each CCD package remove residual field curvature within a single CCD field. 


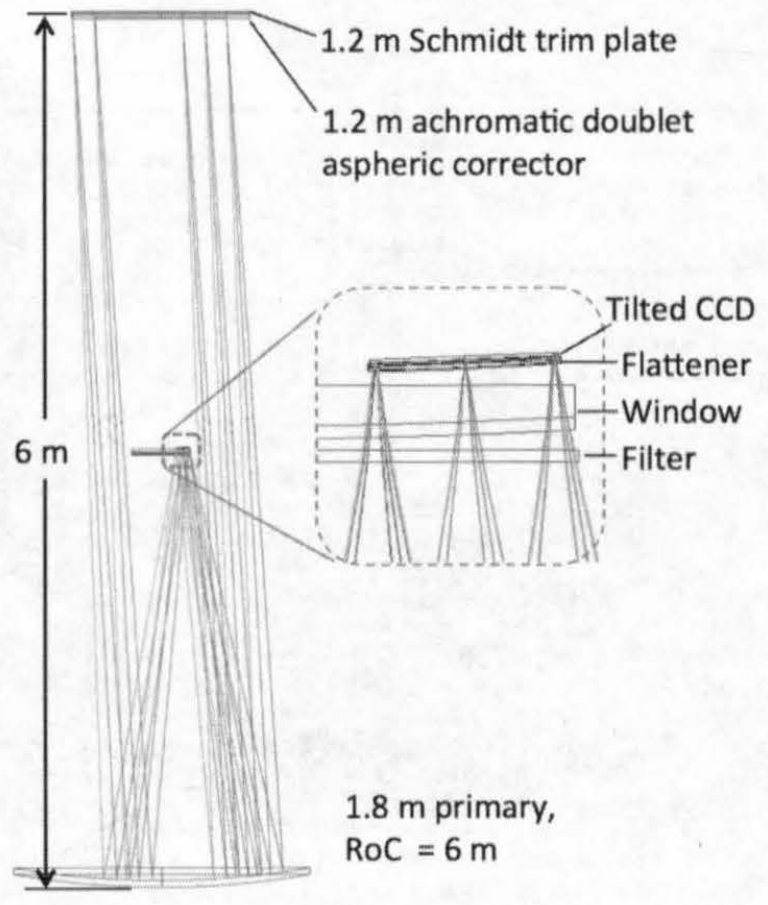

Figure 2: Optical layout for ZTF mounted in Samuel Oschin Telescope.

Table 1: Delivered image quality error budget. The major error terms in the 2 2.2" FWHM filter-dependent goals are natural seeing, the residual aberrations of the field-flattened Schmidt design, and charge diffusion in the CCDs themselves.

ZTF systems engineering has been developing a detailed delivered image quality (DIQ) error budget, shown in Table 1. The DIQ budget includes the contributions (assumed independent) from a number of design, fabrication, and positional tolerancing analyses, with emphasis on the tight positional tolerances imposed by the fast focal length of the Oschin Schmidt design. The assumed seeing is based on a 3year internal site monitoring survey conducted at Palomar Observatory near the 200" telescope, but is believed appropriate for the Oschin Telescope site.

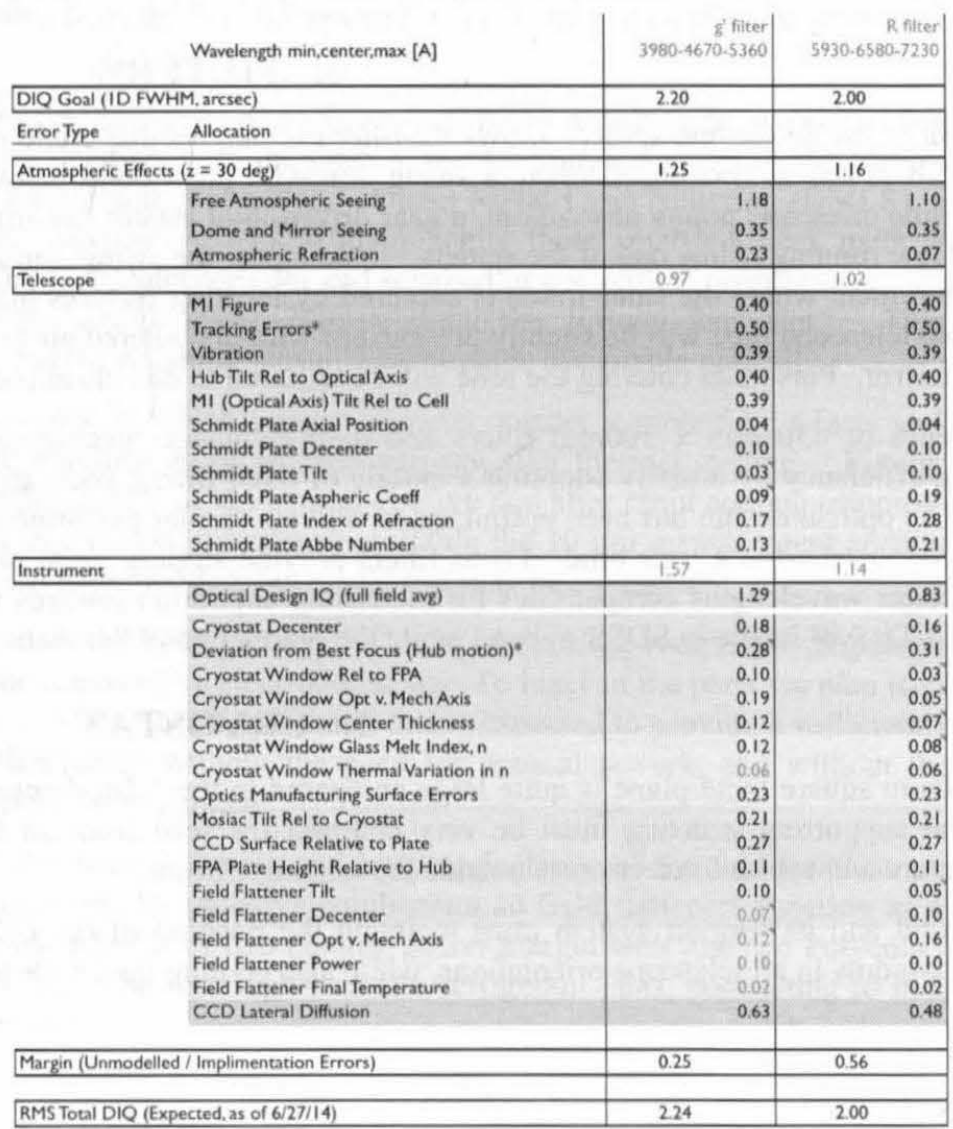




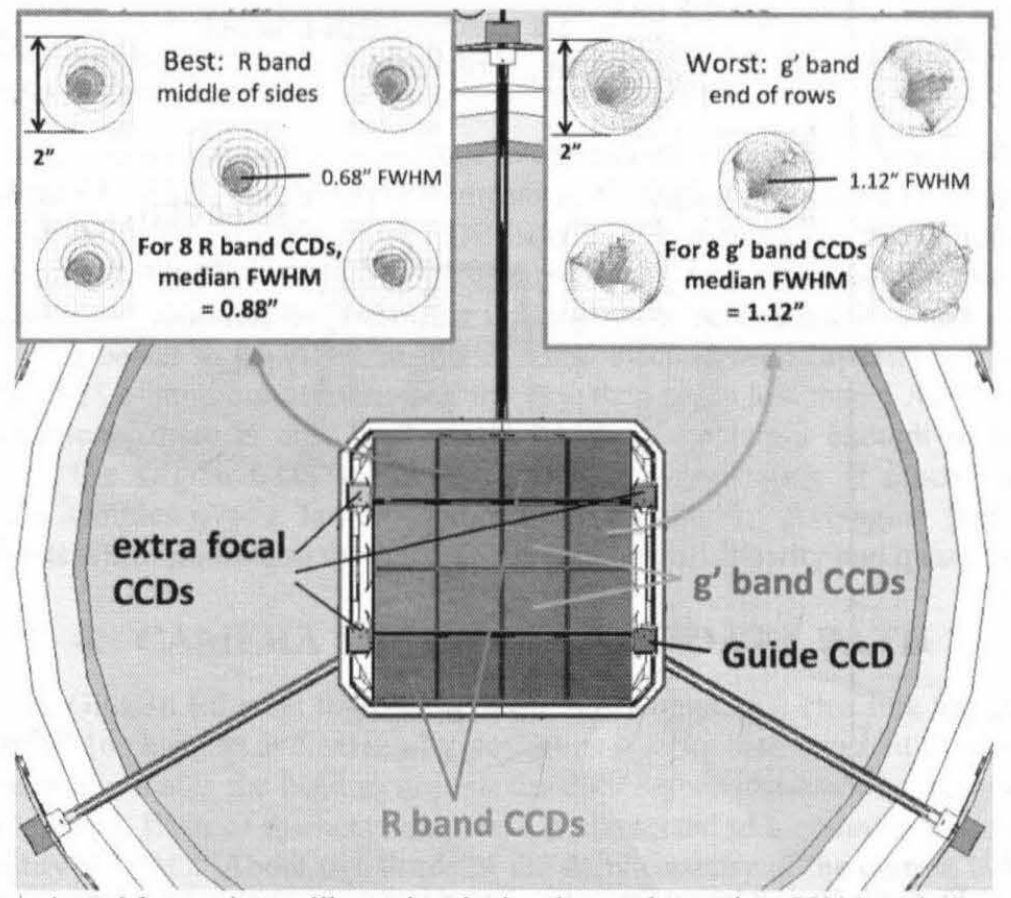

Figure 3: ZTF camera viewed from primary illustrating the low beam obstruction: $20 \%$ by window and $2.5 \%$ by spiders. Insets show spot diagrams for the indicated CCDs, which exhibit best and worst PSFs. For the fixed filter case shown, the outer rows with R filters deliver median $\mathrm{FWHM}=0.88$ ". For inner rows with g' filters, median FWHM $=1.12$ ".

\section{FILTERS}

The layout of the dewar and spiders allows for filter exchangers that could safely stow up to three full size filters. Each of these filters has its own mechanism, a swing arm with pivot point below the outer ends of each of the three spider vanes. While telescope points near zenith, a gear driven joint rotates the arm from a storage location parallel to the side wall to a line running below one of the spiders. In doing so, the swing arm lifts a permanently attached filter to the front of the instrument where the filter-frame is captured by locating features hidden beneath each of the three spider vanes. The closed telescope tube will be slightly pressurized with dry filtered air to prevent dust accumulation on the filters and primary mirror. Personnel entering the tube will be required to don clean room suits and follow clean room protocols.

The expense of $430 \mathrm{~mm} \times 500 \mathrm{~mm}$ filters and their exchange mechanisms may be postponed with modest loss of observing-efficiency by initially adopting a mosaic of fixed filters: coatings applied to the flat side of the field flattener lenses. The optical design has been optimized to delivers similar performance with fixed or exchangeable filters, which can thus be installed at a later time. Fixed filters provide slightly more uniform image quality, since improvement in PSF at longer wavelengths compensates for increasing aberration towards the corners: in the fixed filter case, middle rows of CCDs will image in SDSS g' band while the Northern and Southern rows will image in Mould R band.

\section{CRYOSTAT}

The $\sim 400 \mathrm{~mm}$ square focal plane is quite large compared to the $1.2 \mathrm{~m}$ diameter telescope beam which it obstructs so the dewar and supporting structure must be very compact (as seen from all field angles), while hiding the refrigeration infrastructure and cables to electronics outside the telescope beam.

The cryostat and its support system must maintain the position of the CCDs relative to the curved image plane and powered window in all telescope orientations, while also cooling the CCDs to $\sim 160 \mathrm{~K}$. 


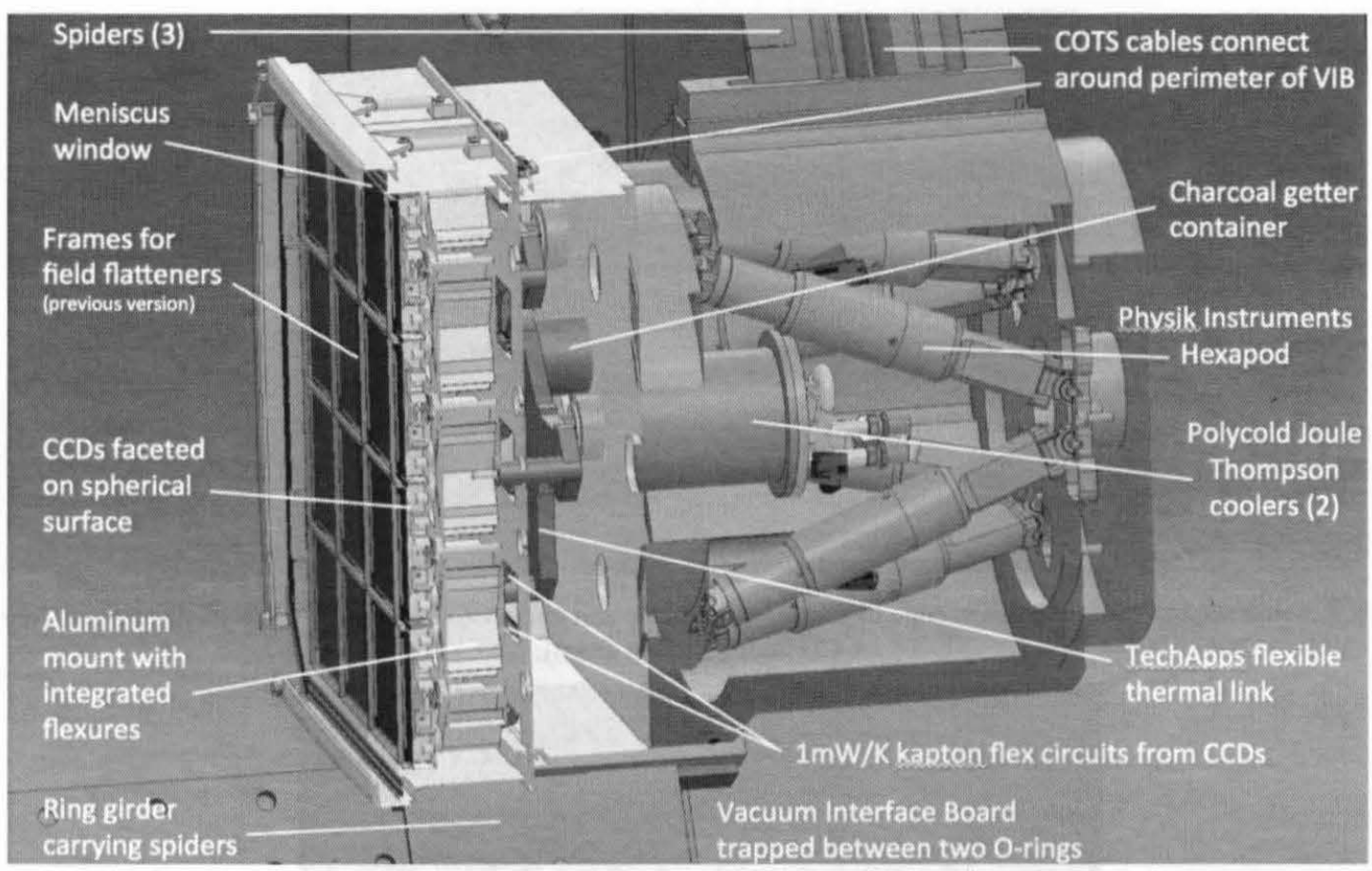

Figure 4: Side view of ZTF Camera showing subtle curvature of CCD mosaic. Note how the dewar becomes progressively smaller towards the rear. Signals are carried to the exterior by the Vacuum Interface Board (green) which is trapped between $\mathrm{O}$-rings at the joint between side wall and rear cover so that no hermetic connectors are required

The dominant thermal load on the CCD cooling system is radiation from the window. Without shielding this would exceed $\sim 70 \mathrm{~W}$. Fortuitously the field flattener lenses can double as floating radiation shields, to almost halve the radiative load from the window. A key technology to support this design is a cost effective way to manufacture the 32 thin and complex thermally isolating frames that hold the field lenses and attach to the sides of the silicon carbide CCD package.

We propose to trap the 16 field flattener lenses between pairs of stainless steel plates attaching to two sides of each CCD package as shown in Figure 5. The plates make contact with the glass at the 45-degree chamfer in the corners of each lens and deform elastically to accommodate differential contraction. Direct Metal Laser Sintering (DMLS), a form of 3D printing, is being investigated, as it is an inexpensive way to manufacture these small complex parts. Prototype stainless steel parts have been manufactured by GPIprototype.com and scanned in our optical profilometer. They show significant but tolerable static deviation form straightness, $\sim 200 \mu \mathrm{m}$ along the $100 \mathrm{~mm}$ lens slot. This can be accommodated in the mechanical tolerancing and the resulting lens displacement is acceptable optically.

A significant concern was that the additive process, in which a layer of metal powder is melted by a laser and cools in place, may result in residual stress that could manifest as significant warpage after thermal cycling. Figure 8 shows profiles along the same path $(+-50 \mu \mathrm{m})$ down the middle of the lens slot before and after rapid cooling (dipping in liquid Nitrogen, warming, repeat 20 times) but no shape change was detected within the $10 \mu \mathrm{m}$ measurement accuracy of the optical profilometer. It appears that this concern is unfounded, but a cold test may be advisable.

We are investigating claims by alternative vendors that they can deliver lower raw surface roughness. Separately we are also testing the ability for the sharp peaks to be removed by electropolishing. To blacken the parts we plan to plate with electroless nickel followed by "black-chrome". After electro-polishing, this is expected to provide a sufficiently smooth surface for direct contact with the fused silica lenses without the need for manual rework, and without the loss of dimensional accuracy that would occur if the parts were painted.

The combination of poor thermal contact to the field flatteners and low conductance of the thin stainless steel struts allows the field flattener temperature to be governed by radiative equilibrium so field flatteners function as a floating radiation shields. As noted, this provides almost a factor-of-two cooling power margin with just two Polycold Compact Coolers with PT30 refrigerant (identical to PTF). The four high-pressure refrigerant lines, connecting to independent compressors, are hidden within the 3rd spider vane. 


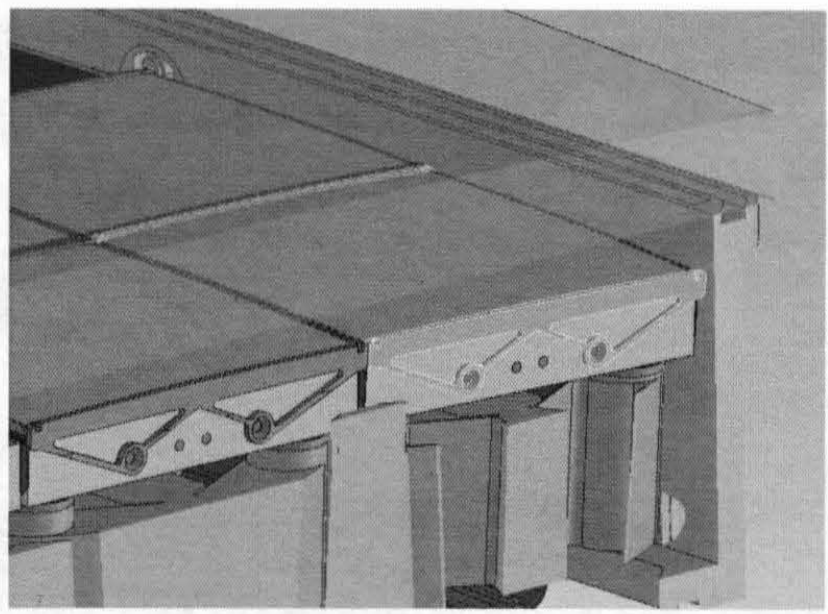

Figure 5: Cross section cutting between edge of science focal plane and guide $\mathrm{CCD}$ to reveal field flattener frames. (corner $\mathrm{CCD}$ highlighted in green).

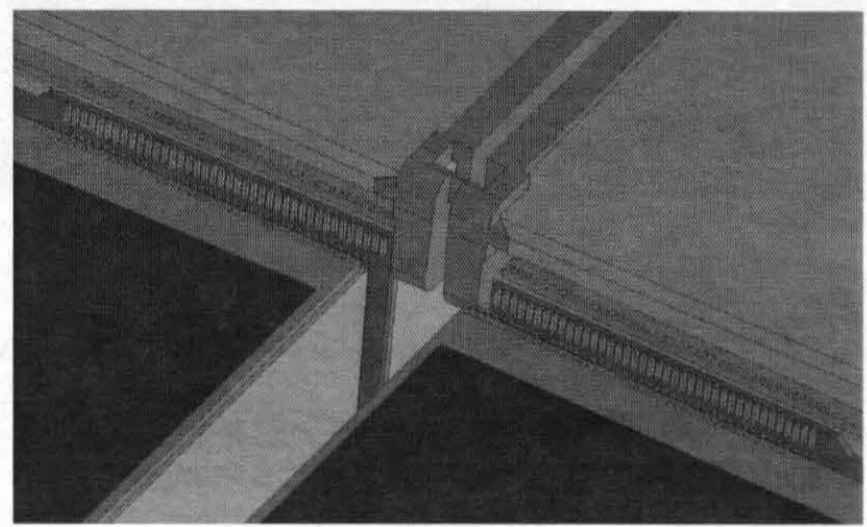

Figure 6: The enlarged view of a corner where $4 \mathrm{CCD}$ come into close proximity is shown with field flatteners removed from CCDs nearest the viewer to reveal the $2 \mathrm{~mm}$ clearance over the bond pads. The frames and lenses prevent contact with the CCD itself.

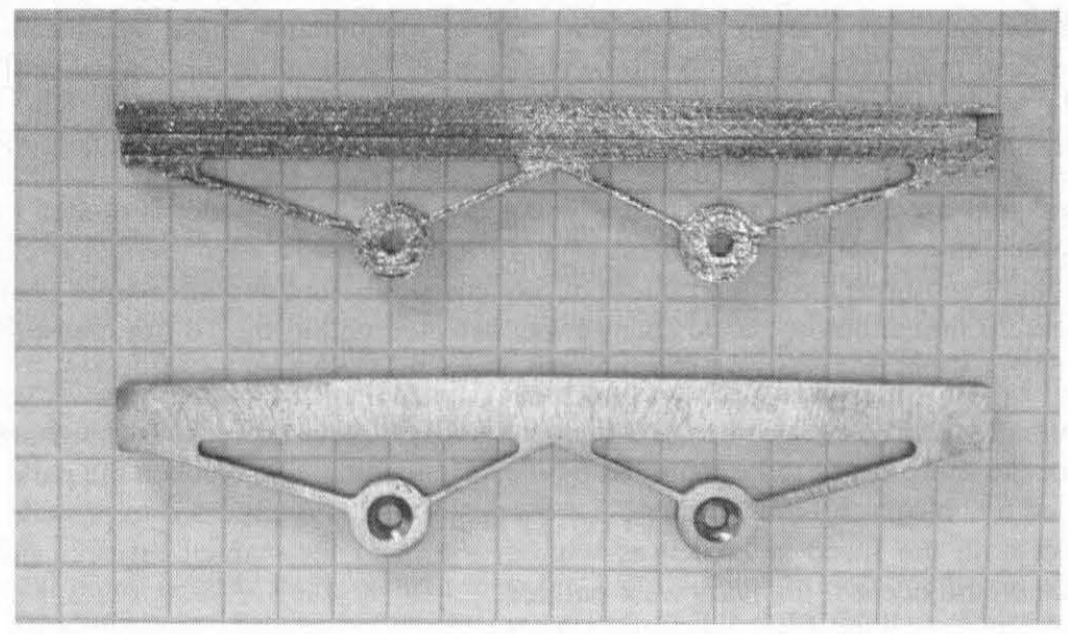

Figure 7: photograph of prototype field lens frames made from stainless steel by GPIprototype.com using DMLS. The lens will be trapped vertically by the slot and located in XY by 45 degree faces at the ends which contact chamfers on the corners of the glass.. (The manufacturer appears to have ground the outer surface by hand when this was not requested.) 


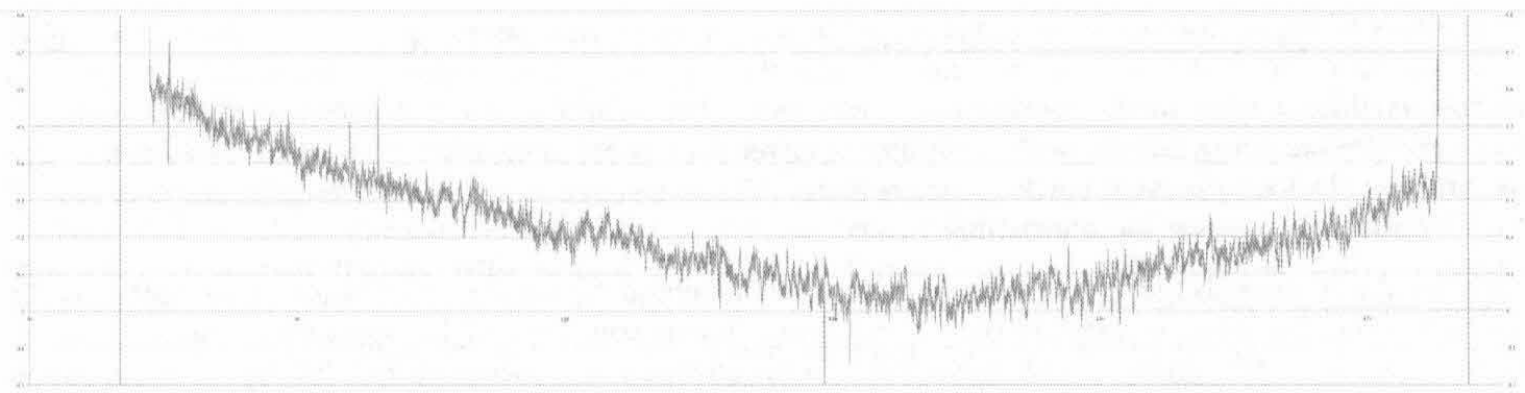

Figure 8: surface profile along bottom of lens pocket before and after 20 rapid thermal cycles for stainless steel prototype made by DMLS: warpage due to stress relief is $<10 \mu \mathrm{m}$. (100 $\mu \mathrm{m}$ per vertical division, $100 \mathrm{~mm}$ horizontal span). The sharpest spikes appear to be bad data points.
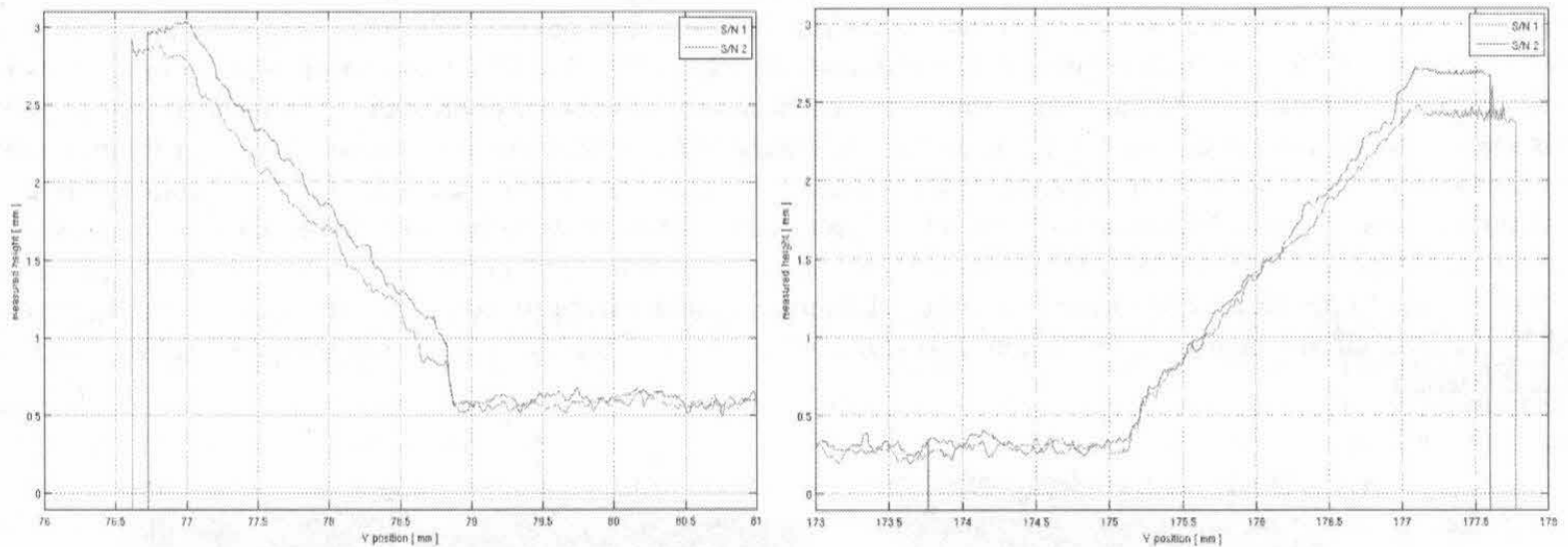

Figure 9: These scans compare the positions of 45 degree faces that define the field lens positions, for two different parts indicating that lens positions may vary $\sim 200 \mu \mathrm{m}$ due to manufacturing variations. (This is acceptable.)

The dewar window is supported by double O-rings at the edge of the optical beam and will be surrounded by a thin frame which must be compliant enough to protect it form impact yet strong enough to support the window in the even that the vacuum is released. Given that the frame must wrap around the spherical shape of the window a 3D printed part made form carbon-filled nylon is under consideration. We have had good experience supporting mirrors in the past with printed parts made by solidconcepts.com from NyTek1200 by Selective Laser Sintering.
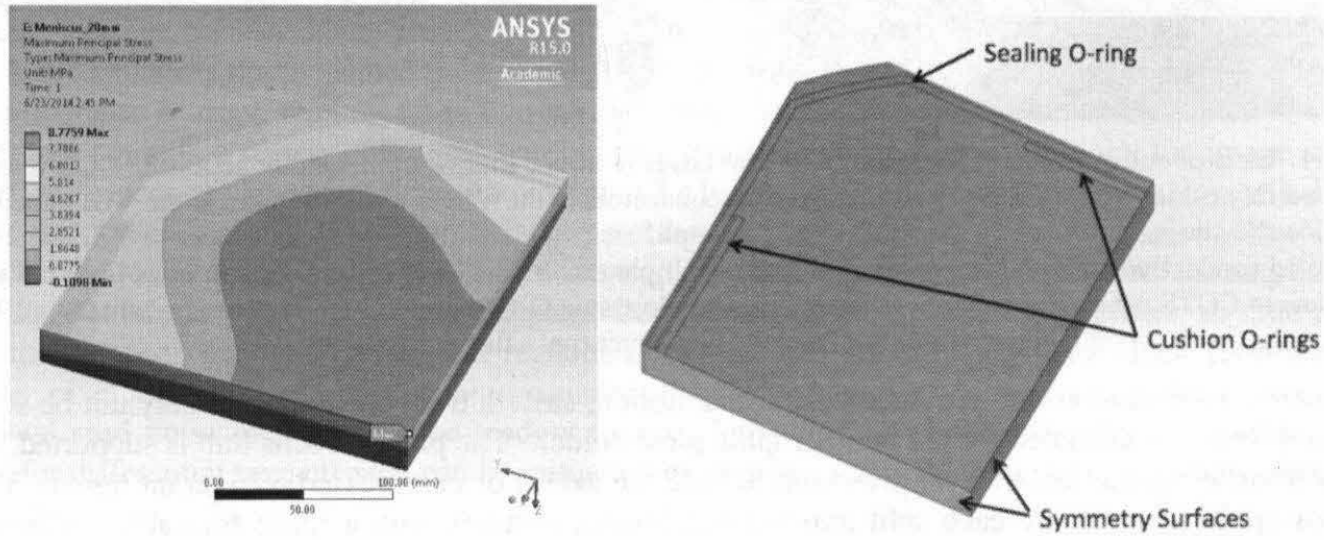

Figure 10: Stress distribution (left) predicted by finite element analysis of one quadrant fused silica window with $28 \mathrm{~mm}$ center thickness, $5674 \mathrm{~mm}$ outer surface radius and $2914 \mathrm{~mm}$ inner surface radius, using elastic supports to represent the gasket pattern shown at right. This view has the CCD side of the window to the top. 
Finite element analysis of the slightly meniscus window predicts a factor of safety equal to six. To achieve this figure the inner O-ring was removed in the corners: this concentrates the supporting forces more in the middle of the sides where the window is thicker (due to the rectangular perimeter). The resulting stress distribution is less centrally concentrated, extending more along the diagonals, resulting in lower peak stress at the center. Vertical faces at the edge of the window will be polished to prevent crack initiation there. Visual inspection and mild over pressure tests will be employed to test for infant mortality prior to installing CCDs.

The dewar width is reduced towards the rear so that it remains hidden behind the window even when viewed off-axis. To achieve $20 \%$ obstruction, the window extends only two O-ring thicknesses beyond the converging beam, and the window frame only extends a few millimeters beyond that. Beam obstruction is increased by $\sim 2 \%$ by the inclusion of $2 \mathrm{~K} \times 2 \mathrm{~K}$ guide and focus CCDs along two edges of the focal plane, but this loss of throughput is expected to be more than compensated by improvement in DIQ made possible by the these sensors.

A major simplification to the dewar wiring is provided by the "Vacuum Interface Board" (VIB). This alternative to hermetic connectors was pioneered in small CCD cameras by Mackay[12], and scaled successfully to 14"x22" by Atwood[13]. The VIB is trapped between O-rings in the sidewall and rear cover as shown in Figure 4 and Figure 11. ZTF's VIB is a 0.125 " thick multilayer printed circuit board, which, at 19.1" x 17.4", fits within the common 24 "x18" size limit for many vendors. While bare resin finish has been shown to make a good seal on other VIBs, gold plated copper is contemplated for lower emissivity, with the secondary benefit of lower outgassing. 860 signals are routed from the CCD connectors on internal layers around cut-outs for access to CCD fasteners and flex cables. 120 spare connector pins are mostly used for additional grounds. Traces continue on internal layers between the two O-rings to 14 non-hermetic through-hole connectors placed around the slightly cantilevered perimeter such that these rear-facing connectors lie close to the side walls of the rear cover. Unreliable hand wiring to expensive hermetic connectors is thus replaced by printed circuit board traces to conventional connectors and highly reliable off the shelf cables made by Samtec and Glenair.
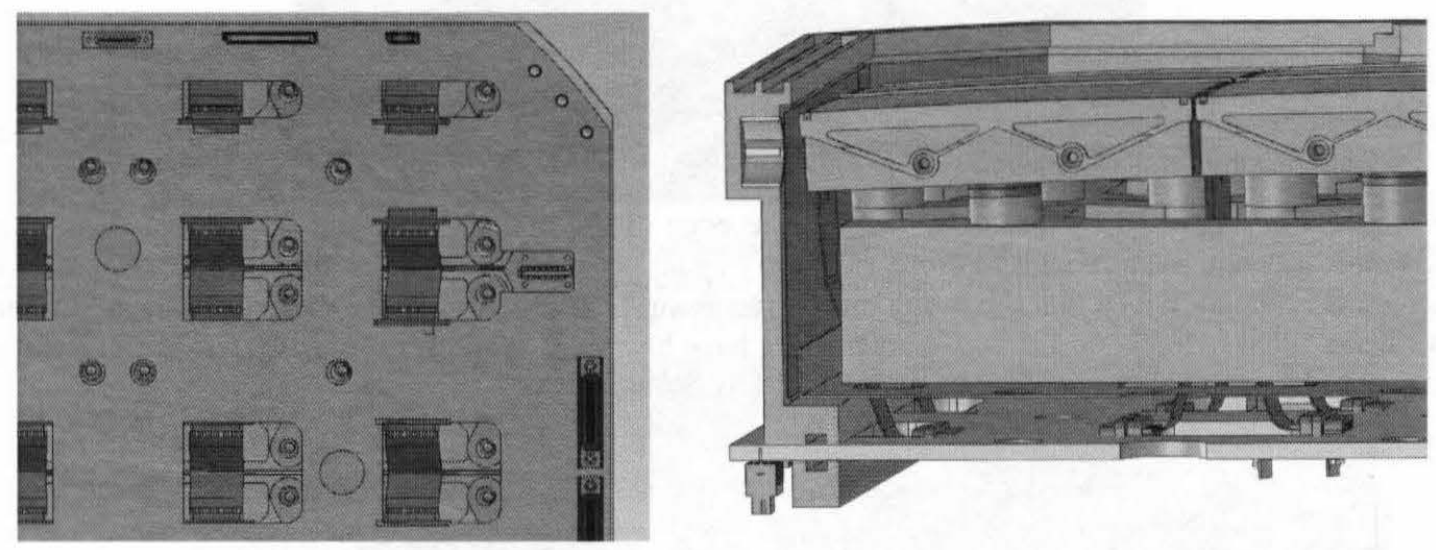

Figure 11: View of one quadrant of VIB (left) with rear cover removed to reveal cut outs for accessing CCD retention hardware and flex cables from CCDs. These engage with connectors in the VIB inserting parallel to the VIB (sideways) on the CCD side shown approximately in the right pane. Preamplifiers (not shown) will be located close to the connectors and will be cooled conductively through copper ground and power planes. Signals will be routed on internal layers of the VIB to connectors to COTS cables located around the perimeter. Opposing O-ring grooves are seen here where the VIB passes between side wall and the rear cover (only flange is shown).

The current instrument-mounting hub provides only focus motion, suffers from backlash and may not be strong enough to carry ZTF as it was only designed for the photographic plate holder. The present focus hub is supported by four solid spiders with uniform cross-section with no provision to hide the cables or compressed refrigerant hoses. These will be replaced by new spiders, which are each split into top and bottom sections with a space for cables in between (at the widest point). The vanes have triangular cross section, tapering towards the outer edges so that their beam obstruction is almost independent of field angle. Profiling of their surface reduces scattered light. Adopting three spiders instead of four reduces the beam obstruction. Diffracted power is also reduced and distributed into 6 spikes in the image instead of four. In combination, these effects will reduce the extent of diffraction around bright stars by a factor of two. 


\section{FOCUS, TILT AND WAVEFRONT SENSING}

A commercial 6-axis hexapod is baselined primarily to provide control of focus, tip and tilt. A variant of the Physik Instrument H-850 will fit in the space and provide large performance margins for load $(250 \mathrm{~kg})$, repeatability $(200 \mathrm{~nm})$ and resolution $(5 \mathrm{~nm})$, so hysteresis and quantization should be negligible. All six degrees of freedom will be utilized: tip/tilt/Z will provide focus across the full field, and compensate for flexure and thermal expansion; XY will maintain centration of faceted mosaic with respect to the curved focal surface; field rotation will correct for polar axis misalignment and differential atmospheric dispersion. The long travel will come into play when mapping surface height or to diagnose optical aberrations as a function of field position at greater spatial resolution than provided by the three $2 \mathrm{~K} \times 2 \mathrm{~K}$ extra focal imaging sensors. Such aberrations might arise from mirror or corrector support problems.

The three-fold symmetry of the hexapod matches that of the three-vane spider to provide a direct mechanical load path. The openings between struts align with 3 spider vanes providing a clear path to route cables to the spiders.

For autofocus capability, we place $2 \mathrm{~K}^{*} 2 \mathrm{~K} 15 \mu \mathrm{m}$ CCDs at 3 'corners' of the focal plane, defocussed by $1.5 \mathrm{~mm}$. We baseline fully depleted n-channel CCDs design by Semiconductor Technology Associates, manufactured by Dalsa and delta-doped and AR coated and packaged by the Micro Devices Laboratory at JPL. The custom package allows for close butting of these focus and guide CCDs along two edges of the science focal plane to squeeze the guiders into the optically corrected field and minimize the growth in instrument size saving a few percent in beam obstruction compared to commercial devices. The fact that these devices have both extended UV and red response is a consequence of their application to other projects but serves to increase the number of photons collected as there are no field flatteners or filters on these CCDs.

The extra-focal imaging method, pioneered by Tokovinin[14] (CTIO) and refined for DES and LSST by Roodman[15] (SLAC) will be used for measuring the low-order Zernike coefficients of the wavefront errors. The focus term at three corners provides an accurate measure of piston/tip/tilt with little sensitivity to image seeing, which is monitored by the $4^{\text {th }}$ (guider) CCD. As for DES and LSST, analysis of the intensity distribution across the defocussed image will also provide a measurement of higher order wavefront errors that can be diagnostic various misalignment or mirror support problems[16]. Extra-focal-imaging CCDs share the science shutter so exposures are concurrent. Readout is pixel and line-synchronous with the science CCDs, but completes in $1 / 3$ rd the time, so that with sufficiently fast analysis the hexapod could be repositioned before the following exposure.

\section{GUIDING}

A similar $2 \mathrm{~K} \times 2 \mathrm{~K}$ CCD located in the $4^{\text {th }}$ "corner will be parfocal with the science CCDs so that it can be used for guiding. This may only prove necessary during the longer exposures used for reference image building, for specialized experiments which are not part of fast cadence survey, or for diagnostics such as settling after slews, tracking errors and wind shake. At 4 megapixels, the guiders are quite large. This and the extended response both to the blue and red, makes chance of finding a bright guide star quite good. This allows the guide CCD to operate in full frame mode and read out while the shutter is open. Since guide stars are bright, read noise is less a concern so $1 \mathrm{MHz}$ pixel rate can be used. With split frame readout, readout takes two seconds, full frame. It is anticipated that after the first $2 \mathrm{~s}$ exposure and $2 \mathrm{~s}$ full frame readout, a region of interest will be automatically selected, at which point the image smear time will drop to tens of milliseconds. Exposure time can remain at $2 \mathrm{~s}$ to integrate the seeing, but smear time will drop to $1 \%$. At the same time that a region of interest is selected, the pixel rate can be reduced to bring read noise below sky noise. The guide and focus CCDs are configured to read from one amplifier per serial register, this allows the amplifier at the other end to be used the reference side of a differential pair. One can still select the nearest amplifier, so little functionality is lost. As for the science channels, differential readout is expected to strongly attenuate clock feedthrough transients enabling faster pixel rate and reducing line start transient (typically a consequence of serial clock feedthrough), at the cost of root-two read noise increase. Since readout time is dominated by parallel for region- of-interest readout, the noise penalty for differential transmission can be mitigated by approximately doubling the pixel time, without significant loss of frame rate.

\section{EXPOSURE SHUTTER}

To avoid severe beam obstruction, the shutter blades must retract to a position outside the $1.2 \mathrm{~m}$ diameter telescope beam. For forty years this was achieved on the Oschin Telescope with curved panels pivoting from a closed position just 
behind the Schmidt corrector, to park against the inner wall of the telescope tube when open. For ZTF, we exploit advances in materials to baseline a bi-parting shutter using flat panels placed just in front of the corrector where, when closed, it additionally protects the corrector optics from contamination. Lightweight panels with carbon fiber face sheet and structural foam core can weigh only $\sim 2 \mathrm{~kg}$ each, yet be resistant to wind loads in excess of $35 \mathrm{mph}$ or accidental loading during cleaning. The blades will be driven in opposite directions by a common belt. Half a second is allocated to open and close, requiring $\sim 2 \mathrm{~g}$ acceleration. Timing stability will far exceed that required for $0.1 \%$ photometric error given that the shutter is close to the pupil and servo controlled. The combination of careful balancing, low moving mass, and programmable velocity profile will reduce telescope shake to negligible levels. The use of flat blades in front of the corrector allows the development of the shutter off-site by the University of Bonn, which has extensive experience developing large custom shutters including the one for DECam. A novel feature is an air extractor to remove dust dislodged from the blades before it falls on the corrector.

\section{TELESCOPE UPGRADES}

To minimize the observing overhead between exposures, the current single-speed dome drive will be replaced by a commercial variable-speed servo motor with shaft encoding for local feedback. The existing bar code reader will continue to provide the outer position control loop. If necessary, a new control algorithm may adjust the velocity profile using pointing information for current and next exposures to take advantage of the dome aperture being oversized.

The telescope currently slews by the full ZTF field width in $13 \mathrm{~s}$ leaving only $2 \mathrm{~s}$ for settling. Additional settling time will be provided on the declination axis by doubling the gear ratio, to gain acceleration at the expense of unneeded maximum speed. Declination is preferred for the fast scan direction since the moment of inertia is lower and this axis is already equipped with a directly-coupled 0.154 " resolution Heidenhain RCN 823 absolute 23 bit encoder. Less frequent stepping in RA opposite to the direction of sidereal motion will minimize airmass.

The baffle between shutter and dome will be redesigned to accommodate the new shutter. The original windscreen may be refurbished to reduce wind loading on both baffle and the enlarged area presented to the wind by the shutter housing.

\section{SOFTWARE UPGRADES}

A new Observatory Control System (OCS) will schedule observations automatically according to a pre-programmed sequence, modified only by dome closure due to adverse weather or Target of Opportunity observations. Although the ZTF targeting system will retain flexibility in support of certain experiments, the increased use of planned observations will allow us to minimize slew time overheads. An important efficiency improvement over the PTF adaptive queue scheduler will be the generation of a target list prior to start of observing instead of PTF's fully real-time dynamic target selection. This is enabled in part by the tremendous increase in ZTF's survey speed, allowing full coverage of the available sky in one night.

The OCS will coordinate the telescope, dome and camera, while the camera software will be responsible for the exposure timing, and subsequent readout by multiple CCD controllers.

Improvements will be made on both sides of the interfaces to telescope and camera to provide more reliable coordination and eliminate delays. To this end a data logger has been installed in PTF to monitor the observatory wide system timing. Communication upgrades will be made to assure that image header information containing pointing and time information is more reliably associated with the correct images than in PTF.

Telescope control software will be re-tuned to minimize slew and settling times after mechanical upgrades to the declination axis. The newly implemented speed control of the dome will be utilized to both improve repointing time and reduce wear. Algorithms will be improved for dome and windscreen control to take advantage of the fact that the dome aperture is oversized and the pointing for the next field is known ahead of time. 


\section{PROJECT STATUS}

ZTF is enabled by a private/public partnership. Early development has been supported by California Institute of Technology, USA; Weizmann Institute for Science, Israel; Oskar Klein Centre at University of Stockholm, Sweden; and Humboldt University, Germany; Lawrence Berkeley National Laboratory, USA; Los Alamos National Laboratory, USA; the Jet Propulsion Laboratory, USA; the University of Wisconsin-Milwaukee, USA; and the TANGO Consortium, Taiwan. At the time of this manuscript preparation, the ZTF collaboration is seeking public support through the US National Science Foundation (NSF) Mid-Scale Instrumentation Program. The collaboration has procured several long lead-time CCDs and developed the camera cryostat to the detailed design level. With full funding, we expect the first light of ZTF in late 2016, with the start of science operations beginning in early 2017.

\section{REFERENCES}

[1] Law, N., Kulkarni, S., Dekany, R., "The Palomar Transient Factory: System Overview, Performance, and First Results," PASP, 121, 1395L (2009).

[2] Levi, M., Bebek, C., Beers, T., Blum, R., Cahn, R., Eisenstein, D., Flaugher, B., Honscheid, K., Kron, R., Lahav, O., McDonald, P., Roe, N., Schlegel, D., representing the DESI collaboration, "The DESI Experiment: a white paper for Snowmass 2013," eprint arXiv:1308.0847.

[3] Minkowski, R. L., \& Abell, G. O., [Stars and Stellar Systems, Vol. 3], Basic Astronomical Data, ed. K. A. Strand, Chicago University Press, 481 (1963).

[4] Downing, M., Baade, D., Deiries, S., Jorden, P., "Bulk Silicon CCDs, Point Spread Functions, and Photon Transfer Curves: CCD Testing Activities at ESO" Detectors for Astronomy, (2009).

http://www.eso.org/sci/meetings/2009/dfa2009/program.html

[5] Bredthauer, G., "Archon: A modern controller for high performance astronomical CCDs," Proc. SPIE 9147, 9147202 (2014).

[6] Gach, J-L., Darson, D., Guillanume, C., Goillandeau, M., Cavadore, C., Balard, P., Boissin, O., Boulesteix, J., “A new digital CCD readout technique for Ultra-Low-Noise CCDs" PASP vol. 115, 1068--1071 (2003)

[7] Onaka, P., et al.,"GPC1 and GPC2: The Pan-STARRS 1.4 gigapixel mosaic focal plane CCD cameras with an onsky on-CCD tip-tilt image compensation", SPIE 8453, 8453J-1 (2012)

[8] Clapp, M. J., "Development of a test system for the characterisation of DCDS CCD readout techniques," Proc. SPIE 8453, 8453D-1 (2012).

[9] Harris, R. D., Jorden, P. R., Bastable, M., pike, A., Dryer, M., Pittock, R., Marin-Fanch, A., Taylor, K., Palmer, I., Wheeler, P., Renshaw, R., Fenemore-Jones, G., Taylor, A., Haddow, G., Swindels, I., Barwick, M., “A gigapixel cryogenic focal plane array camera for JPAS $2.5 \mathrm{~m}$ survey telescope," Proc. SPIE 9154, 915428 (2014)

[10] Smith, R. M., Kaye, S., "Fully differential signal path for the ZTF mosaic," Proc. SDW2013 (2013)

[11] Smith, R. M., Kaye, S., "Digital correlated double sampling for ZTF," Proc. SDW2013 (2013)

[12] Mackay, C. "High-Speed, Photon Counting CCD cameras for Astronomy", ESO Workshop on Scientific Detectors, Garching, (October 2009). http://www.eso.org/sci/meetings/2009/dfa2009/program.html

[13] Atwood, B., Jorden, P., "The KMTNet 340 Megapixel focal planes," Proc. SDW2013 (2013).

[14] Tokovinin, A., Heathcote, S., "Donut: measuring optical aberrations from a single extra-focal image," PASP Vol. 118, No. 846. 1165-1175 (August 2006). doi:10.1086/506972

[15] Roodman, A. J., Riel, K. A., Davis, C., "Wavefront sensing and the active optics system of the dark energy camera," Proc. SPIE 9145, 914541 (2014)

[16] Schechter, P. L., Sobel Levinson, R., "Generic misalignment aberration patterns and the subspace of benign misalignment," Proc. SPIE 8444, 844455 (2012). http://dx.doi.org/10.1117/12.925075 